\title{
Produção de cachos e parâmetros genéticos de híbridos de caiaué com dendezeiro
}

\author{
Ricardo Lopes ${ }^{(1)}$, Raimundo Nonato Vieira da Cunha( ${ }^{(1)}$ e Marcos Deon Vilela de Resende(2)
}

\begin{abstract}
(1)Embrapa Amazônia Ocidental, Rodovia AM 010, Km 29, Caixa Postal 319, CEP 69010-970 Manaus, AM. E-mail: ricardo.lopes@embrapa.br, raimundo.cunha@embrapa.br ${ }^{(2)}$ Embrapa Florestas, Estrada da Ribeira, Km 111, Caixa Postal 319, CEP $83411-000$ Colombo, PR. E-mail: deon@cnpf.embrapa.br
\end{abstract}

\begin{abstract}
Resumo - O objetivo deste trabalho foi avaliar a produção de cachos e estimar os parâmetros genéticos de progênies $\mathrm{F} 1$ de caiaué (Elaeis oleifera) com dendezeiro (E. guineenses). As progênies foram plantadas em parcelas com número variável de plantas. Avaliaram-se número (NC), peso médio (PMC) e peso total de cachos (PTC) por planta, do sétimo ao décimo-terceiro ano após o plantio. Estes dados foram analisados por REML/BLUP em 59 progênies, com delineamento desbalanceado em linha e coluna. Todos os caracteres apresentaram considerável variabilidade, com coeficientes de variação genética entre 18,1 e 25,5\%, quanto à progênie, e de 25,6 a 36,0\% quanto a indivíduo. Pela seleção das cinco melhores progênies, podem ser obtidos ganhos superiores a $30 \%$, para NC e PTC, e acima de $20 \%$ para PMC; e, com a seleção dos cinco melhores indivíduos, ganhos acima de $65 \%$, para NC e PTC, e acima de 60\% para PMC. As progênies apresentam alta variabilidade genética quanto à produção de cachos. Ganhos genéticos elevados podem ser obtidos pela seleção tanto de indivíduos para clonagem quanto de progênies para reprodução sexuada.
\end{abstract}

Termos para indexação: Elaeis guineensis, Elaeis oleifera, palma de óleo africana, palma de óleo americana, REML/BLUP, repetibilidade, seleção simultânea.

\section{Bunch yield and genetic parameters of progenies between caiaué and African oil palm}

\begin{abstract}
The objective of this work was to evaluate the bunch yield and to estimate the genetic parameters of F1-progenies between caiaué (Elaeis oleifera) and African oil palm (E. guineenses). The progenies were planted in plots with a variable number of plants. The number of bunches (NB), average bunch weight (ABW), and fresh fruit-bunch yield (FFB) were evaluated, per plant, from the seventh to the thirteenth year after planting. These data were analyzed by REML/BLUP in 59 progenies, with a row-and-column unbalanced design. All characters showed a considerable genetic variability, with genetic variation coefficients between 18.1 and $25.5 \%$ for progenies, and 25.6 and $36.0 \%$ for individuals. Gains of more than $30 \%$, for NB and FFB, and above $20 \%$ for ABW can be obtained selecting the five best progenies. By selecting the five best individuals, gains of more than $65 \%$ for NB and FFB, and above $60 \%$ for ABW, can be obtained. The progenies show a high-genetic variability for bunch yield. High-genetic gains can be obtained by selecting both for individuals, for clonal propagation, and for progenies, for seed propagation.
\end{abstract}

Index terms: Elaeis guineensis, Elaeis oleifera, African palm oil, American palm oil, REML/BLUP, repeatability, simultaneous selection.

\section{Introdução}

O dendezeiro, ou palma de óleo africana (Elaeis guineensis Jacq.), é a principal fonte mundial de óleo vegetal. Em 2010, a produção mundial de óleo de palma, em 14,99 milhões de hectares de área cultivada, foi de 45,09 milhões de toneladas, enquanto a produção de óleo de soja, em 102,38 milhões de hectares, foi de 39,76 milhões de toneladas (Food and Agriculture Organization fo the United Nations, 2012). Além do potencial produtivo da espécie, o custo de produção do óleo de palma é inferior ao das principais oleaginosas
(Zimmer, 2009); por isso, esta espécie é comumente considerada fundamental para que se possa atender a crescente demanda mundial por óleo vegetal que, estima-se, deverá ser de 240 milhões de toneladas em 2050 (Corley, 2009).

No Brasil, a partir do Zoneamento Agroecológico para palma de óleo (ZAE-Palma de óleo), foram identificados aproximadamente 30 milhões de hectares aptos para o cultivo do dendezeiro somente nas áreas já desmatadas da Amazônia Legal, (Ramalho Filho et al., 2010). Isto indica que o país possui a maior área

Pesq. agropec. bras., Brasília, v.47, n.10, p.1496-1503, out. 2012 
mundial disponível para expansão da cultura. Porém, a expectativa de expansão da dendeicultura, não somente no Brasil, mas em toda a América Latina, é ameaçada por uma anomalia de etiologia ainda desconhecida, denominada amarelecimento-fatal. Identificada há mais de 30 anos, essa anomalia já dizimou milhares de hectares de dendezeiro (De Franqueville, 2003).

Apesar dos estudos realizados nas últimas décadas, ainda não se encontrou um método de controle eficaz do amarelecimento-fatal, mas sabe-se que o caiaué, ou palma de óleo americana [E. oleifera (H.B.K.) Cortes], e seu híbrido com o dendezeiro não são afetados pela anomalia (Barcelos et al., 2002; Cunha et al., 2012). Apesar das semelhanças com a espécie africana, o óleo do caiaué, espécie de domesticação incipiente(Clement, 1999), tem produtividade inferior à das cultivares utilizadas em plantios comerciais de dendezeiro, resultantes do melhoramento sistemático da espécie, que vem sendo realizado há, aproximadamente, um século. Entretanto, o caiaué apresenta características vantajosas em relação ao dendezeiro, como menor taxa de crescimento vertical do tronco - que lhe confere menor porte e reduz custo de colheita -, óleo mais insaturado e resistência ou tolerância a diversas pragas e doenças que hoje acometem a espécie africana (Cunha et al., 2012).

$\mathrm{Na}$ América do Sul, o Brasil e a Colômbia têm conduzido os principais programas de melhoramento genético do dendezeiro que utilizam o caiaué (Barcelos et al., 2000; Rey et al., 2004; Bastidas et al., 2007). Há grande variabilidade a ser explorada no caiaué (Barcelos et al., 2002; Rios et al., 2011), e suas combinações interespecíficas com o dendezeiro precisam ser avaliadas. Acredita-se ser possível desenvolver cultivares interespecíficas tão produtivas como as cultivares atuais de dendezeiro, introduzir resistência ou tolerância a pragas e doenças, tornar o óleo mais insaturado e reduzir o crescimento da planta.

O peso total de cachos por planta é o principal determinante da produtividade em dendezeiro, por isso é essencial para os programas de melhoramento genético conhecer o controle genético dessa característica (Okwuagwu et al., 2008; Okoye et al., 2009). Estimativas de parâmetros genéticos e correlações entre os caracteres associados à produção de cachos já foram realizadas em diferentes populações de dendezeiro (Soh et al., 2003; Okwuagwu, 2008; Ortega Cedillo et al., 2008; Okoye et al., 2009), mas ainda não existem informações sobre essas estimativas em populações híbridas interespecíficas entre o caiaué e o dendezeiro. O procedimento BLUP tem sido usado no melhoramento do dendezeiro, tanto para a seleção de genitores (Soh, 1994) quanto para a seleção de cruzamentos (Purba et al., 2001).

O objetivo deste trabalho foi avaliar a produção de cachos e estimar parâmetros genéticos de progênies híbridas interespecíficas entre caiaué (Elaeis oleifera) e (E. guineenses) dendezeiro, por meio da metodologia RELM/BLUP.

\section{Material e Métodos}

O experimento foi realizado no Campo Experimental do Rio Urubu, da Embrapa Amazônia Ocidental, no Município de Rio Preto da Eva, AM. O clima, segundo a classificação de Köppen, é do tipo Ami, quente e úmido, com valores médios de temperatura máxima de $31,2^{\circ} \mathrm{C}$, e temperatura mínima de $23,5^{\circ} \mathrm{C}$, e com precipitação anual em torno de $2.200 \mathrm{~mm}$. O solo é um Latossolo Amarelo, de textura muito argilosa (Guillaumet et al., 2003).

Foram avaliadas 59 progênies F1 entre o caiaué e o dendezeiro, plantadas em 1991, em parcelas com número variável de plantas por progênie (Tabela 1). O bloco experimental consistiu de 19 linhas, com média de 31 plantas, formando uma área retangular de aproximadamente $140 \times 270 \mathrm{~m}$. Cada progênie foi representada por uma parcela, estabelecida com a divisão do número de plantas da progênie em duas linhas paralelas. A avaliação do número e peso dos cachos foi realizada do sétimo ao décimo terceiro ano após o plantio, perfazendo sete anos consecutivos. Foram realizadas colheitas quinzenais durante o período de avaliação.

Os valores anuais de número de cachos (NC), peso médio (PMC) e total de cachos (PTC) de cada indivíduo foram analisados por meio da metodologia de modelos lineares mistos (REML/BLUP), com delineamento desbalanceado em linha e coluna. Como o bloco experimental era retangular - formado por uma grade determinada por filas no sentido das linhas e das colunas (posição da planta na linha), com controle de genealogia - aplicou-se a análise genética REML/BLUP com ajuste para os efeitos ambientais nos sentidos das linhas e das colunas e, também, para a tendência ambiental no gradiente diagonal. Esse procedimento 
Tabela 1. Identificação genealógica de 59 híbridos interespecíficos F1 de caiaué com dendezeiro, plantados em 1991, em parcelas com número variável de plantas por progênie.

\begin{tabular}{|c|c|c|c|c|c|}
\hline \multirow[t]{2}{*}{ Descendência } & \multicolumn{2}{|c|}{ Genealogia materna } & \multicolumn{2}{|c|}{ Genealogia paterna } & \multirow{2}{*}{$\begin{array}{l}\text { Plantas por } \\
\text { progênie }\end{array}$} \\
\hline & Genitor & Origem & Genitor & Origem & \\
\hline & \multicolumn{2}{|c|}{ Dendezeiro } & \multicolumn{2}{|r|}{ Caiaué } & \\
\hline LM 15451 & L 4867 D & 15 D x M269 D & CAI 09 & Caimbé (Tefé) & 14 \\
\hline LM 15133 & LM 3323 D & D x DA 3 D & CAI $110 \mathrm{D}$ & Caimbé (Tefé) & 10 \\
\hline LM 15291 & LM $4790 \mathrm{D}$ & $69 \mathrm{D} \times \mathrm{DA} 115 \mathrm{D}$ & CAI $110 \mathrm{D}$ & Caimbé (Tefé) & 5 \\
\hline \multirow[t]{2}{*}{ LM 15412} & LM 6193 D & D x DA 115 D & CAI $81 \mathrm{D}$ & Caimbé (Tefé) & 2 \\
\hline & \multicolumn{2}{|c|}{ Caiaué } & \multicolumn{2}{|r|}{ Dendezeiro } & \\
\hline RUB 36 & CAI 33 D & Caimbé (Tefé) & LM 3035 D & Deli - DA 681 & 12 \\
\hline RUB 43 & CAC $35 \mathrm{D}$ & Manicoré & LM 3035 D & Deli - DA 681 & 12 \\
\hline RUB 47 & CAC 34 D & Manicoré & LM 3038 D & Deli - DA 681 & 11 \\
\hline RUB 52 & CAC $32 \mathrm{D}$ & Manicoré & LM 3038 D & Deli - DA 681 & 10 \\
\hline RUB 63 & CAC 33 D & Manicoré & LM 3038 D & Deli - DA 681 & 11 \\
\hline RUB 38 & CAI 133 D & Caimbé (Tefé) & LM 2937 D & Deli - LM 1053 & 13 \\
\hline RUB 55 & CAC 37 D & Manicoré & LM $2550 \mathrm{D}$ & Deli - LM 1053 & 10 \\
\hline RUB 61 & CAC $32 \mathrm{D}$ & Manicoré & LM $2550 \mathrm{D}$ & Deli - LM 1053 & 8 \\
\hline RUB 71 & CAC $42 \mathrm{D}$ & Manicoré & LM $2550 \mathrm{D}$ & Deli - LM 1053 & 10 \\
\hline RUB 48 & CAC $31 \mathrm{D}$ & Manicoré & LM 2937 D & Deli - LM 1053 & 11 \\
\hline RUB 67 & CAC $23 \mathrm{D}$ & Manicoré & LM 2937 D & Deli - LM 1053 & 9 \\
\hline RUB 45 & CAC $35 \mathrm{D}$ & Manicoré & LM 2940 D & Deli - LM 1053 & 10 \\
\hline RUB 54 & CAC $51 \mathrm{D}$ & Manicoré & LM 2940 D & Deli - LM 1053 & 10 \\
\hline RUB 58 & CAC $48 \mathrm{D}$ & Manicoré & LM 2940 D & Deli - LM 1053 & 12 \\
\hline RUB 64 & CAC 24 D & Manicoré & LM 2940 D & Deli - LM 1053 & 5 \\
\hline RUB 51 & CAC $25 \mathrm{D}$ & Manicoré & LM $5003 \mathrm{~T}$ & LM10T AF - LM 2764 & 10 \\
\hline RUB 59 & CAC $49 \mathrm{D}$ & Manicoré & LM $5003 \mathrm{~T}$ & LM10T AF - LM 2764 & 11 \\
\hline RUB 62 & CAC $33 \mathrm{D}$ & Manicoré & LM $5003 \mathrm{~T}$ & LM10T AF - LM 2764 & 12 \\
\hline RUB 80 & CAC $51 \mathrm{D}$ & Manicoré & $1114 \mathrm{P}$ & LM2T AF - CEPLAC & 10 \\
\hline RUB 81 & CAC $45 \mathrm{D}$ & Manicoré & $1114 \mathrm{P}$ & LM2T AF - CEPLAC & 10 \\
\hline RUB 82 & CAC $18 \mathrm{D}$ & Manicoré & $1114 \mathrm{P}$ & LM2T AF - CEPLAC & 9 \\
\hline RUB 84 & CAC 23 D & Manicoré & $1114 \mathrm{P}$ & LM2T AF - CEPLAC & 10 \\
\hline RUB 89 & RU 20 D & Manicoré & $1114 \mathrm{P}$ & LM2T AF - CEPLAC & 12 \\
\hline RUB 93 & RU 19 D & Manicoré & $1114 \mathrm{P}$ & LM2T AF - CEPLAC & 12 \\
\hline RUB 90 & RU $21 \mathrm{D}$ & Manicoré & $1114 \mathrm{P}$ & LM2T AF - CEPLAC & 12 \\
\hline RUB 33 & CAI $84 \mathrm{D}$ & Caimbé (Tefé) & LM $2052 \mathrm{~T}$ & LM2T AF - LM 495 & 11 \\
\hline RUB 44 & CAC 29 D & Manicoré & LM $2032 \mathrm{~T}$ & LM2T AF - LM 495 & 12 \\
\hline RUB 53 & CAC $49 \mathrm{D}$ & Manicoré & LM $2032 \mathrm{~T}$ & LM2T AF - LM 495 & 4 \\
\hline RUB 56 & CAC $48 \mathrm{D}$ & Manicoré & LM $2032 \mathrm{~T}$ & LM2T AF - LM 495 & 12 \\
\hline RUB 65 & CAC 22 D & Manicoré & LM $2032 \mathrm{~T}$ & LM2T AF - LM 495 & 12 \\
\hline RUB 68 & CAC $28 \mathrm{D}$ & Manicoré & LM $2032 \mathrm{~T}$ & LM2T AF - LM 495 & 10 \\
\hline RUB 40 & CAC $27 \mathrm{D}$ & Manicoré & LM $2052 \mathrm{~T}$ & LM2T AF - LM 495 & 9 \\
\hline RUB 92 & RU 14 D & BR 174 & $5820-07 \mathrm{P}$ & LM2T AF - LM 725 & 9 \\
\hline RUB 72 & CAC $51 \mathrm{D}$ & Manicoré & $5820-07 \mathrm{P}$ & LM2T AF - LM 725 & 10 \\
\hline RUB 73 & CAC $45 \mathrm{D}$ & Manicoré & 5820-07 P & LM2T AF - LM 725 & 9 \\
\hline RUB 75 & CAC 29 D & Manicoré & 5820-07 P & LM2T AF - LM 725 & 10 \\
\hline RUB 76 & CAC $21 \mathrm{D}$ & Manicoré & 5820-07 P & LM2T AF - LM 725 & 5 \\
\hline RUB 77 & CAC 23 D & Manicoré & 5820-07 P & LM2T AF - LM 725 & 10 \\
\hline RUB 85 & CAC 23 D & Manicoré & 5820-07 P & LM2T AF - LM 725 & 10 \\
\hline RUB 86 & CAC $19 \mathrm{D}$ & Manicoré & 5820-07 P & LM2T AF - LM 725 & 10 \\
\hline RUB 87 & CAC $19 \mathrm{D}$ & Manicoré & 5820-07 P & LM2T AF - LM 725 & 11 \\
\hline RUB 88 & CAC $46 \mathrm{D}$ & Manicoré & 5820-07 P & LM2T AF - LM 725 & 11 \\
\hline RUB 91 & RU 16 D & BR $174-\mathrm{Km} 500$ & 5820-07 P & LM2T AF - LM 725 & 10 \\
\hline RUB 79 & CA $20 \mathrm{D}$ & UEPAE - Manaus & $5820-07 \mathrm{P}$ & LM2T AF - LM 725 & 10 \\
\hline RUB 34 & CAI $22 \mathrm{D}$ & Caimbé (Tefé) & LM $6219 \mathrm{~T}$ & LM431T x LM319P - LM 5629 & 6 \\
\hline RUB 35 & CAI $27 \mathrm{D}$ & Caimbé (Tefé) & LM $6223 \mathrm{~T}$ & LM431T x LM319P - LM 5629 & 9 \\
\hline RUB 50 & CAC 27 D & Manicoré & LM $6219 \mathrm{~T}$ & LM431T x LM319P - LM 5629 & 10 \\
\hline RUB 60 & CAC $47 \mathrm{D}$ & Manicoré & LM $6219 \mathrm{~T}$ & LM431T x LM319P - LM 5629 & 12 \\
\hline RUB 42 & CAC 19 D & Manicoré & LM $6223 \mathrm{~T}$ & LM431T x LM319P - LM 5629 & 12 \\
\hline RUB 57 & CAC $48 \mathrm{D}$ & Manicoré & LM $6223 \mathrm{~T}$ & LM431T x LM319P - LM 5629 & 12 \\
\hline RUB 66 & CAC 42 D & Manicoré & LM $6919 \mathrm{~T}$ & LM431T x LM319P - LM 5629 & 12 \\
\hline RUB 37 & CAI $62 \mathrm{D}$ & Caimbé (Tefé) & LM $3673 \mathrm{~T}$ & Nigéria - NIFOR WA 11 & 5 \\
\hline RUB 41 & CAC 34 D & Manicoré & LM $3673 \mathrm{~T}$ & Nigéria - NIFOR WA 11 & 11 \\
\hline RUB 39 & CAC 29 D & Manicoré & LM $3673 \mathrm{~T}$ & Nigéria - NIFOR WA 11 & 10 \\
\hline RUB 46 & CAC 27 D & Manicoré & LM $3673 \mathrm{~T}$ & Nigéria - NIFOR WA 11 & 10 \\
\hline
\end{tabular}

Pesq. agropec. bras., Brasília, v.47, n.10, p.1496-1503, out. 2012 
permite estimação eficiente de parâmetros genéticos para seleção. Os efeitos de linhas e colunas foram considerados como aleatórios, para que fosse possível recuperar a informação genética entre linhas e entre colunas. Assim, os parâmetros genéticos foram estimados via REML e os valores genotípicos (ou médias genotípicas ajustadas de progênies), genéticos aditivos e genotípicos individuais foram estimados pelo procedimento BLUP, por meio do pragrama Selegen REML/BLUP (Resende, 2002).

Para a avaliação genética, utilizou-se o modelo estatístico $\mathrm{y}=\mathrm{Xm}+\mathrm{Zg}+\mathrm{Wp}+\mathrm{Tl}+\mathrm{Hc}+\varepsilon$, em que $\mathrm{y}, \mathrm{m}, \mathrm{g}, \mathrm{p}, \mathrm{l}, \mathrm{c}$, e $\varepsilon$ são, respectivamente, vetores de dados de efeitos: de medições (fixos); genotípicos de progênies de irmãos germanos (aleatório); permanentes de indivíduos (aleatório); de linha (aleatórios); de coluna (aleatórios); e de erros aleatórios. As matrizes de incidência são X, Z, W, T, H, para m, g, p, 1 e c, respectivamente.

Para obter as estimativas das herdabilidades individuais no sentido restrito, foram utilizadas duas abordagens: uma considerou a ausência de dominância, ou mais exatamente, negligenciou $25 \%$ da variação genética de dominância que está presente na variação genotípica entre progênies; e outra considerou a presença de dominância completa em uma população com nível intermediário de melhoramento; nesse caso, a variação genética de dominância equivaleu à metade da variação genética aditiva (Vencovsky, 1987).

Na seleção de progênies superiores, consideraramse, simultaneamente, os caracteres NC, PTC e PMC e aplicou-se o índice de seleção baseado na média dos rankings de cada progênie nos três caracteres.

\section{Resultados e Discussão}

Todos os caracteres apresentaram considerável variabilidade genética, com coeficientes de variação genética de 18,1 a 25,5\%, quanto às progênies, e de 25,6 a $36,0 \%$ quanto aos indivíduos, o que indica excelentes possibilidades para a seleção (Tabela 2). As estimativas das herdabilidades individuais no sentido restrito, em que se considerou a ausência de dominância, foram de 30,6, 31,6 e 38,0\% para os caracteres PMC, PTC e NC, respectivamente. Quando se considerou a presença de dominância completa, as herdabilidades individuais no sentido restrito foram de 24,5, 25,3 e 30,4\%, para os caracteres PMC, PTC e NC, respectivamente. Considerando-se ambos os extremos, ou seja, a ausência e a presença de dominância completa, verificou-se que as herdabilidades apresentaram pequena variação, com ótimas magnitudes em ambos os casos, o que confirma as excelentes possibilidades para a seleção. Soh et al. (2003) relatam herdabilidades de 0,22 a 0,36 para esses caracteres em dendezeiro.

As estimativas de repetibilidade, quando estimadas simultaneamente às herdabilidades no sentido restrito, são muito importantes na aferição dos reais graus de dominância. Isto porque a repetibilidade impõe um limite superior no valor da herdabilidade individual no sentido amplo (Falconer, 1987). No presente trabalho, as estimativas de repetibilidade equivaleram a 50, 40 e $40 \%$ para os caracteres PMC, PTC e NC, respectivamente. Assim, os valores da herdabilidade individual no sentido amplo não podem ultrapassar esses valores. Ao se considerar dominância completa, tais herdabilidades apresentaram estimativas equivalentes a 36,75, 37,91 e $45,66 \%$, respectivamente. Portanto, o pressuposto de controle genético via dominância completa é invalido para o caráter $\mathrm{NC}$, uma vez que ultrapassou o limite de $40 \%$ determinado na análise de repetibilidade. Mesmo quando se considerou dominância parcial baixa grau médio de dominância igual a 0,25 - o valor da herdabilidade no sentido amplo ultrapassou o valor de $40 \%$. Assim, tal caráter pode ser considerado como

Tabela 2. Estimativas de parâmetros genéticos e fenotípicos quanto ao número de cachos (NC), peso total de cachos (PTC) e peso médio de cachos (PMC) de progênies interespecíficas F1 de caiaué com dendezeiro.

\begin{tabular}{lccc}
\hline Parâmetro & NC & PTC & PMC \\
\hline Variância genotípica entre progênies & 3,24 & 554,28 & 5,92 \\
Variância permanente de indivíduo & 3,45 & 808,66 & 11,04 \\
Variância entre linhas & 0,02 & 32,64 & 2,38 \\
Variância entre colunas & 0,08 & 4,20 & 0,04 \\
Variância ambiental & 10,22 & $2.108,77$ & 19,28 \\
Variância fenotípica & 17,00 & $3.508,55$ & 38,67 \\
$\mathrm{R}^{2}$ de progênie & $0,19 \pm 0,02$ & $0,16 \pm 0,02$ & $0,15 \pm 0,02$ \\
$\mathrm{R}^{2}$ de efeitos permanentes de indivíduo. & 0,20 & 0,23 & 0,29 \\
$\mathrm{R}^{2}$ de linhas & 0,0009 & 0,0093 & 0,0616 \\
$\mathrm{R}^{2}$ de colunas & 0,0046 & 0,0012 & 0,0010 \\
HAI sem dominância & 0,3805 & 0,3160 & 0,3062 \\
HAI individual com dominância completa & 0,3044 & 0,2528 & 0,2450 \\
HGI individual com dominância completa & 0,4566 & 0,3791 & 0,3675 \\
Repetibilidade individual & $0,40 \pm 0,03$ & $0,40 \pm 0,03$ & $0,50 \pm 0,03$ \\
CV genotípica de progênie (\%) & 25,5 & 23,7 & 18,1 \\
CV genética aditiva individual (\%) & 36,0 & 33,5 & 25,6 \\
\hline Média geral & 7,06 & 99,30 & 13,46 \\
\hline
\end{tabular}

$\mathrm{R}^{2}$, coeficiente de determinação. HAI, herdabilidade aditiva individual. HGI, herdabilidade genotípica individual. CV, coeficiente de variação. 
herdado aditivamente, com herdabilidade individual no sentido restrito igual a $38 \%$. Purba et al. (2001) relataram não haver significância para os efeitos de dominância nos caracteres produtivos avaliados em dendezeiro. Um resultado comum entre os trabalhos mencionados e o presente é a alta herdabilidade para o caráter número de cachos (maior que $35 \%$ ) e a consequente baixa dominância. Isto justificaria os ganhos que se obtêm com a seleção recorrente recíproca em dendezeiro (Soh et al., 2009), esquema este que conduz ao aumento da heterose, a qual, por sua vez, só ocorre se houver dominância alélica (Falconer \& Makay, 1996).

As estimativas dos coeficientes de determinação entre progênies e dos efeitos permanentes de indivíduos dentro de progênies apresentaram valores similares para NC, e mais discrepantes para PTC e PMC (Tabela 2). Isto é coerente com o maior grau de dominância associado a esses dois últimos caracteres, pois, segundo Vencovsky \& Barriga (1992), a variação genotípica entre progênies contempla metade da variância genética aditiva e um quarto da variação genética de dominância, ao passo que a variação dos efeitos permanentes de indivíduos dentro de progênies contempla metade da variância genética aditiva, três quartos da variação genética de dominância e mais a variação de ambiente permanente. Assim, quanto maior o nível de dominância, maior deve ser o coeficiente de determinação dos efeitos permanentes de indivíduos dentro de progênies, em relação ao coeficiente de determinação entre progênies.

As estimativas do coeficiente de determinação dos efeitos de coluna foram praticamente iguais a zero (Tabela 2), o que evidencia que não havia heterogeneidade ambiental a ser corrigida, no sentido das colunas, na área experimental. No entanto, a estimativa do coeficiente de determinação dos efeitos de linha mostrou-se considerável $(6.2 \%)$ para o caráter de menor herdabilidade (PMC), o que evidencia ter havido heterogeneidade ambiental a ser corrigida, no sentido das linhas, na área experimental.

Com o aumento do número de avaliações em cada planta, ocorreram aumentos da determinação fenotípica permanente, da determinação genética aditiva de indivíduo e também da acurácia genética, o que aumenta a eficiência da seleção (Tabela 3). Com as sete avaliações (anos), as herdabilidades em nível individual aumentaram para 78 (NC), 65 (PTC) e 53\% (PMC), o que propiciou acurácias seletivas de $88,5,80,7$ e $73,1 \%$, respectivamente, para a seleção de indivíduos. Os valores obtidos são uma indicação de que a seleção de indivíduos, com o número de avaliações realizadas, terá grande eficiência.

As estimativas obtidas com o aumento do número de medições também permitiram determinar o número mínimo de anos de avaliação necessário para a seleção com diferentes valores de eficiência (Tabela 3). Para o caráter NC, verifica-se que com três anos é possível fazer seleção com acurácia de $80 \%$. No entanto, a eficiência da seleção baseada em determinado número de anos, em relação à seleção baseada em um só ano, aumenta consideravelmente em até cinco anos (eficiência de 1,39, contra eficiência de 1,29, para quatro anos). Após cinco anos, o ganho em eficiência é praticamente desprezível para todos os caracteres. Para o caráter de menor herdabilidade (PMC), o uso de cinco anos é necessário para se atingir acurácia de $71 \%$. Assim,

Tabela 3. Estimativas de determinação fenotípica permanente e determinação genética aditiva, acurácia seletiva e eficiência do uso de determinado número de anos de avaliação, em relação ao uso de apenas um ano, para número, peso total e peso médio de cachos de híbridos interespecíficos de caiaué com dendezeiro.

\begin{tabular}{|c|c|c|c|c|}
\hline Anos & $\begin{array}{c}\text { Determinação } \\
\text { fenotípica }\end{array}$ & $\begin{array}{c}\text { Determinação } \\
\text { genética }\end{array}$ & $\begin{array}{c}\text { Acurácia } \\
\text { seletiva }\end{array}$ & Eficiência \\
\hline \multicolumn{5}{|c|}{ Número de cachos } \\
\hline 1 & 0,40 & 0,38 & 0,62 & 1,00 \\
\hline 2 & 0,57 & 0,54 & 0,74 & 1,20 \\
\hline 3 & 0,67 & 0,63 & 0,80 & 1,29 \\
\hline 4 & 0,73 & 0,69 & 0,83 & 1,35 \\
\hline 5 & 0,77 & 0,73 & 0,86 & 1,39 \\
\hline 6 & 0,80 & 0,76 & 0,87 & 1,41 \\
\hline 7 & 0,82 & 0,78 & 0,88 & 1,43 \\
\hline \multicolumn{5}{|c|}{ Peso total de cachos $(\mathrm{kg})$} \\
\hline 1 & 0,40 & 0,32 & 0,56 & 1,00 \\
\hline 2 & 0,57 & 0,45 & 0,67 & 1,20 \\
\hline 3 & 0,67 & 0,53 & 0,73 & 1,29 \\
\hline 4 & 0,73 & 0,57 & 0,76 & 1,35 \\
\hline 5 & 0,77 & 0,61 & 0,78 & 1,39 \\
\hline 6 & 0,80 & 0,63 & 0,79 & 1,41 \\
\hline 7 & 0,82 & 0,65 & 0,81 & 1,44 \\
\hline \multicolumn{5}{|c|}{ Peso médio de cachos (kg) } \\
\hline 1 & 0,50 & 0,31 & 0,55 & 1,00 \\
\hline 2 & 0,67 & 0,41 & 0,64 & 1,15 \\
\hline 3 & 0,75 & 0,46 & 0,68 & 1,22 \\
\hline 4 & 0,80 & 0,49 & 0,70 & 1,26 \\
\hline 5 & 0,83 & 0,51 & 0,71 & 1,29 \\
\hline 6 & 0,86 & 0,52 & 0,72 & 1,31 \\
\hline 7 & 0,88 & 0,53 & 0,73 & 1,32 \\
\hline
\end{tabular}


cinco anos parece ser um número adequado para todos os caracteres avaliados, o que propicia determinação fenotípica permanente com valor próximo de $80 \%$.

Ganhos consideráveis podem ser obtidos com a seleção de progênies híbridas para os três caracteres (Tabela 4). Para NC e PTC, ganhos acima de 30\% podem ser obtidos com a seleção das cinco melhores progênies, das quais quatro são coincidentes nos dois caracteres. A correlação genética estimada entre essas duas variáveis foi de 0,84 , o que mostra que NC é um grande determinante da PTC. Correlações positivas entre NC e PTC, de 0,68 a 0,86 , também foram encontradas nas populações de palma de óleo avaliadas por Okwuagwu et al. (2008), e de 0,65 na população avaliada por Okoye et al. (2009). Para o caráter PMC, ganhos genéticos acima de $20 \%$ podem ser obtidos com a seleção das cinco melhores progênies. Entretanto, nenhuma das progênies selecionadas para PMC coincidiu com as cinco melhores para NC e PTC. A correlação genética estimada entre PMC e NC foi de 0,06 , e entre PMC e PTC foi de 0,52. Em relação à correlação entre NC e PMC, Okwuagwu et al. (2008) encontraram valores negativos significativos entre $-0,22$ e - 0,37 , e Okoye et al. (2009) correlação negativa significativa de $-0,57$. Correlações positivas entre PMC e PTC, de 0,18 a 0,24, foram encontradas nas populações de palma de óleo avaliadas por Okwuagwu et al. (2008), e de 0,21 na população avaliada por Okoye et al. (2009).

As cinco melhores progênies na avaliação simultânea dos três caracteres foram: RUB37, RUB40, RUB90, RUB56 e RUB93, com rankings médios de 6,0, 6,7, $8,7,12,7$ e 13,3, respectivamente. Dessas, quatro estão entre as cinco melhores para NC e PTC. A melhor progênie na avaliação simultânea foi a RUB37, que está ordenada nos seguintes postos: 5, 2 e 11 para NC, PTC e PMC, respectivamente.

A seleção dos cinco melhores indivíduos com base em seus valores genéticos aditivos, obtidos pelo procedimento BLUP individual, resultou em ganhos genéticos estimados superiores a $82 \%$ para NC, 64\% para PTC e 52\% para PMC (Tabela 5). Esses ganhos são maiores do que os estimados para a seleção de progênies, e evidenciam o potencial de seleção desses indivíduos para utilização em retrocruzamentos.

Tanto a herdabilidade entre progênies quanto a herdabilidade dentro de progênies contiveram, em seus numeradores, metade da variância genética aditiva mais um quarto da variação genética de dominância. O numerador exato para as duas herdabilidades deveria conter apenas metade da variância genética aditiva. No entanto, como ambas também contiveram um quarto da variação genética de dominância no numerador, a proporcionalidade entre as duas herdabilidades não foi afetada e nem, portanto, o ranking de indivíduos.

Ganhos genéticos elevados podem ser obtidos com a seleção de indivíduos com base em seus valores genotípicos para o caráter peso total de cachos (Tabela 6), com o objetivo de propagação clonal para plantios comerciais. Os ganhos genéticos com a clonagem dos cinco melhores indivíduos, para peso total de cachos, variaram de 71,6 a 99,8\%, o que indica grande vantagem sobre a seleção de progênies, para as quais os ganhos genéticos com a seleção das cinco melhores progênies variaram de 34 a 55\% (Tabela 4). Quando se considera a densidade de plantio de 143 plantas por hectare (Cunha \& Lopes, 2010) e as médias baseadas nos sete anos de avaliação da PTC, as produtividades anuais de cachos desses indivíduos foram de: 26,1 $\mathrm{Mg} \mathrm{ha}^{-1}$ (ind 272 - RUB90), 26,9 $\mathrm{Mg} \mathrm{ha}^{-1}$ (ind 144 - RUB40), 27,0 $\mathrm{Mg} \mathrm{ha}^{-1}$ (ind 511 - RUB37), 27,9 $\mathrm{Mg} \mathrm{ha}^{-1}$ (ind 145 - RUB40) e 31,6 $\mathrm{Mg} \mathrm{ha}^{-1}$ (ind $512-$ RUB37).

Tabela 4. Valores genotípicos e ganho genético com a seleção quanto ao número, peso médio e peso total de cachos das cinco melhores progênies F1 de caiaué com dendezeiro.

\begin{tabular}{lcc}
\hline Progênie & Valores genotípicos & Ganho genético (\%) \\
\hline & \multicolumn{2}{c}{ Número de cachos } \\
RUB90 & 10,9 & 55,04 \\
RUB68 & 10,1 & 43,08 \\
RUB40 & 9,7 & 37,04 \\
RUB80 & 9,7 & 36,95 \\
RUB37 & 9,4 & 33,89 \\
\hline & Peso total de cachos $(\mathrm{kg})$ \\
RUB90 & 150,4 & 51,36 \\
RUB37 & 144,6 & 45,59 \\
RUB40 & 141,3 & 42,23 \\
RUB68 & 128,9 & 29,81 \\
RUB56 & 127,8 & 28,61 \\
\hline & Peso médio de cachos $(\mathrm{kg})$ \\
LM15412 & 18,9 & 40,80 \\
LM15451 & 18,2 & 35,37 \\
RUB79 & 17,8 & 32,15 \\
LM15291 & 16,3 & 21,42 \\
RUB58 & 16,2 & 20,37 \\
\hline
\end{tabular}


Tabela 5. Valores genéticos aditivos individuais e ganho genético com a seleção quanto ao número, peso total e peso médio de cachos, baseada na média de sete anos, dos cinco melhores indivíduos entre 59 progênies híbridas interespecíficas de caiaué com dendezeiro.

\begin{tabular}{lcccc}
\hline Indivíduo & Família & $\begin{array}{c}\text { Valor fenotípico } \\
\text { médio }\end{array}$ & $\begin{array}{c}\text { Valor genético } \\
\text { aditivo }\end{array}$ & $\begin{array}{c}\text { Ganho genético } \\
(\%)\end{array}$ \\
\hline 572 & Número de cachos \\
333 & RUB68 & 14,57 & 13,60 & 92,54 \\
102 & RUB80 & 14,42 & 13,05 & 88,70 \\
512 & RUB37 & 14,00 & 12,74 & 85,92 \\
308 & RUB90 & 13,14 & 12,56 & 83,93 \\
\hline \multicolumn{5}{c}{ Peso total de cachos (kg) } \\
512 & RUB37 & 221,28 & 183,03 & 82,39 \\
145 & RUB40 & 194,85 & 167,97 & 84,27 \\
511 & RUB37 & 189,00 & 165,56 & 69,11 \\
272 & RUB90 & 182,71 & 164,67 & 66,68 \\
144 & RUB40 & 188,00 & 163,15 & 65,79 \\
\hline \multicolumn{5}{c}{ Peso médio de cachos (kg) } \\
542 & LM15412 & 28,16 & 22,96 & 64,26 \\
31 & RUB79 & 27,43 & 21,65 & 70,67 \\
150 & LM15451 & 26,97 & 21,49 & 60,88 \\
165 & RUB52 & 32,57 & 21,47 & 59,75 \\
148 & LM15451 & 24,05 & 20,44 & 59,60 \\
\hline \multicolumn{5}{c}{51,92} \\
\hline 5
\end{tabular}

Tabela 6. Valores genotípicos individuais e ganho genético com a seleção quanto ao peso total de cachos $(\mathrm{kg}$ por ano), baseados na média de sete anos dos cinco melhores indivíduos, entre 59 progênies híbridas interespecíficas F1 de caiaué com dendezeiro.

\begin{tabular}{lcccc}
\hline Indivíduo & Família & $\begin{array}{c}\text { Valor fenotípico } \\
\text { médio }\end{array}$ & $\begin{array}{c}\text { Valor } \\
\text { genotípico }\end{array}$ & $\begin{array}{c}\text { Ganho genético } \\
(\%)\end{array}$ \\
\hline 512 & RUB37 & 221,28 & 198,40 & 99,75 \\
145 & RUB40 & 194,85 & 178,65 & 79,86 \\
511 & RUB37 & 189,00 & 173,94 & 75,12 \\
144 & RUB40 & 188,00 & 171,91 & 73,07 \\
272 & RUB90 & 182,71 & 170,40 & 71,56 \\
\hline
\end{tabular}

\section{Conclusões}

1. As progênies avaliadas apresentam alta variabilidade genética quanto à produção de cachos.

2. É possível obter altos ganhos genéticos com a seleção tanto de indivíduos, para propagação vegetativa, como de progênies, para propagação sexuada.

3. Cinco anos de avaliação da produção de cachos na fase adulta são necessários para se obter acurácia de seleção superior a $80 \%$.

\section{Referências}

BARCELOS, E.; AMBLARD. P.; BERTHAUD, J.; SEGUIN, M. Genetic diversity and relationship in American and African oil palm as revealed by RFLP and AFLP molecular markers. Pesquisa Agropecuária Brasileira, v.37, p.1105-1114, 2002.

BARCELOS, E.; NUNES, C.D.M.; CUNHA, R.N.V. Melhoramento genético e produção de sementes comerciais de dendezeiro. In: VIEGA, I. de J.M.; MULLER, A.A. (Ed.). A cultura do dendezeiro na Amazônia brasileira. Belém: Embrapa Amazônia Oriental; Manaus: Embrapa Amazônia Ocidental, 2000. p.145-174.

BASTIDAS, S.; PEÑA, E.; REYES, R.; PÉREZ, J.; TOLOSA, W. Comportamiento agronómico del cultivar híbrido RC1 de palma de aceite (Elaeis oleifera x Elaeis guineensis) x Elaeis guineensis. Revista Corpoica - Ciencia y Tecnología Agropecuaria, v.8, p.5-11, 2007.

CLEMENT, C.R. 1492 and the loss of Amazonian crop genetic resources. I. The relation between domestication and human population decline. Economic Botany, v.53, p.188-202, 1999.

CORLEY, R.H.V. How much palm oil do we need? Environmental Science and Policy, v.12, p.134-139, 2009.

CUNHA, R.N.V. da; LOPES, R. BRS Manicoré: híbrido interespecífico entre o caiaué e o dendezeiro africano recomendado para áreas de incidência de amarelecimento-fatal. Manaus: Embrapa Amazônia Ocidental, 2010. 4p. (Embrapa Amazônia Ocidental. Comunicado técnico, 85).

CUNHA, R.N.V. da; LOPES, R.; ROCHA, R.N.C. da; LIMA, W.A.A. de; TEIXEIRA, P.C.; BARCELOS, E.; RODRIGUES, M.R.L; RIOS, S.A. Domestication and breeding of the American oil palm. In: BORÉM, A.; LOPES, M.T.G.; CLEMENT, C.R.; NODA, H. (Ed.). Domestication and breeding: Amazonian species. Viçosa: UFV, 2012. p.275-296.

DE FRANQUEVILLE, H. Oil palm rot in Latin American: review paper. Experimental Agriculture, v.39, p.225-240, 2003.

FALCONER, D.S. Introdução à genética quantitativa. Viçosa: UFV, 1987. 279p.

FALCONER, D.S.; MACKAY, T.F.C. Introduction to quantitative genetics. $4^{\text {th }}$ ed. Edinburgh: Longman, 1996. 462p.

FOOD AND AGRICULTURE ORGANIZATION OF THE UNITED NATIONS. FAOSTAT: production crops 2010. Available at: <http://faostat.fao.org/site/567/DesktopDefault. aspx?PageID=567\#ancor $>$. Accessed on: 3 Apr. 2012.

GUILLAUMET, J.L.; RODRIGUES, M.R.L.; MIRANDA, I.P.A. A estação experimental do Rio Urubu - Embrapa Amazônia Ocidental. In: MIRANDA, I.P. de A.; GUILLAUMET, J.L.; BARBOSA, E.M.; RODRIGUES, M. do R.L.; SILVA, M.F.F. da (Ed.). Ecossistemas florestais em áreas manejadas na Amazônia. Manaus: Instituto Nacional de Pesquisas da Amazônia, 2003. p.30-64.

OKOYE, M.N.; OKWUAGWU, C.O.; UGURU, M.I. Population improvement for fresh fruit bunch yield and yield components in oil palm (Elaeis guineensis Jacq.). American-Eurasian Journal of Scientific Research, v.4, p.59-63, 2009. 
OKWUAGWU, C.O.; OKOYE, M.N.; OKOLO, E.C.; ATAGA, C.D.; UGURU, M.I. Genetic variability of fresh fruit bunch yield in Deli/dura x tenera breeding populations of oil palm (Elaeis guineensis Jacq.) in Nigeria. Journal of Tropical Agriculture, v.46, p.52-57, 2008.

ORTEGA CEDILLO, D.S.; BARROS, W.S.; FERREIRA, F.M.; DIAS, L.A. Correlation and repeatability in progenies of African oil palm. Acta Scientiarum. Agronomy, v.30, p.197-201, 2008.

PURBA, A.R.; FLORI, A.; BAUDOUIN, L.; HAMON, S. Prediction of oil palm (Elaeis guineensis, Jacq.) agronomic performances using the best linear unbiased predictor (BLUP). Theoretical and Applied Genetics, v.102, p.787-792, 2001.

RAMALHO FILHO, A.; MOTTA, P.E.F. da; NAIME, U.J.; GONCALVES, A.O.; TEIXEIRA, W.G. Zoneamento agroecológico para a cultura da palma de óleo nas áreas desmatadas da Amazônia Legal. In: RAMALHO FILHO, A.; MOTTA, P.E.F. da; FREITAS, P.L. de; TEIXEIRA, W.G.T. Zoneamento agroecológico, produção e manejo para a cultura da palma de óleo na Amazônia. Rio de Janeiro: Embrapa Solos, 2010. p.57-68.

RESENDE, M.D.V. de. Genética biométrica e estatística no melhoramento de plantas perenes. Brasília: Embrapa Informação Tecnológica; Colombo: Embrapa Florestas, 2002. 975p.

REY, L.; GÓMEZ, P.L.; AYALA, I.; DELGADO, W.; ROCHA, P. Colecciones genéticas de palma de aceite Elaeis guineensis (Jacq.) y Elaeis oleifera (H.B.K) de Cenipalma: Características de importancia para el sector palmicultor. Palmas, v.25, p.39-48, 2004.

RIOS, S. de A.; CUNHA, R.N.V. da; LOPES, R.; BARCELOS, E.; TEIXEIRA, P.C.; LIMA, W.A.A. de; ABREU, S.C. Caracterização fenotípica e diversidade genética em subamostras de caiaué (Elaeis oleifera). Unimontes Científica, v.13, p.50-56, 2011.

$\mathrm{SOH}$, A.C. Ranking parents by best linear unbiased prediction (BLUP) breeding values in oil palm. Euphytica, v.76, p.13-21, 1994.

SOH, A.C.; GAN, H.H.; WONG, G.; HOR, T.Y.; TAN C.C. Estimates of within family genetic variability for clonal selection in oil palm. Euphytica, v.133, p.147-163, 2003.

SOH, A.C.; WONG, C.K.Y.; HO, W.; CHOONG, C.W. Oil palm. In: VOLLMANN, J.; RAJCAN, I. (Ed.). Handbook of plant breeding: oil crops. New York: Springer, 2009. p.333-367.

VENCOVSKY, R. Herança quantitativa. In: PATERNIANI, E.; VIEGAS, G.P. (Ed.). Melhoramento e produção de milho. 2.ed. Campinas: Fundação Cargill, 1987. v.1, p.137-214.

VENCOVSKY, R.; BARRIGA, P. Genética biométrica no fitomelhoramento. Ribeirão Preto: Sociedade Brasileira de Genética, 1992. 486p.

ZIMMER, Y. Cost competitiveness of major oilseeds versus palm oil. 2009. Available at: <http://www.agribenchmark.org/ fileadmin/freefiles/cc_team_publications/Zimmer_oilseeds_2009. pdf $>$. Accessed on: 26 Mar. 2012.

$\overline{\text { Recebido em } 20 \text { de abril de } 2012 \text { e aprovado em } 6 \text { de setembro de } 2012}$ 